\title{
Customized Application of tDCS for Clinical Rehabilitation in Alzheimer's Disease
}

\author{
Claudia Rodella ${ }^{1 *}$, Jesús Cespón ${ }^{2}$, Claudia Repetto ${ }^{1}$ and Maria Concetta Pellicciari ${ }^{3}$ \\ ${ }^{1}$ Department of Psychology, Catholic University of Sacred Heart, Milan, Italy, ${ }^{2}$ Basque Center on Cognition, Brain and \\ Language, San Sebastian, Spain, ${ }^{3}$ UniCamillus-Saint Camillus International University of Health Sciences, Rome, Italy
}

Keywords: tDCS, neurorehabiliation, Alzheimer's disease, customized treatment, cognitive impairment, neuromodulation, cognitive reserve, brain reserve

\section{INTRODUCTION}

Alzheimer's disease (AD) is a neurodegenerative disorder characterized by cognitive-behavior deficits, which strongly impact daily-life activities (Weintraub et al., 2012). Currently, the limited efficacy of pharmacological treatments has encouraged researchers to develop nonpharmacological interventions, such as cognitive training and non-invasive brain stimulation (NIBS) treatments, designed to prevent or delay cognitive impairment (Cass, 2017; Cespón et al., 2018).

In recent years, there has been growing interest in evaluating the use of NIBS to improve

OPEN ACCESS

Edited by:

Suelen Lucio Boschen,

Mayo Clinic, United States

Reviewed by:

Davide Cappon,

Harvard University, United States

Francesca Mameli,

IRCCS Ca 'Granda Foundation

Maggiore Policlinico Hospital, Italy

*Correspondence:

Claudia Rodella

claudia.rodella@unicatt.it

Specialty section:

This article was submitted to

Brain Health and Clinical

Neuroscience,

a section of the journal

Frontiers in Human Neuroscience

Received: 30 March 2021

Accepted: 30 June 2021

Published: 29 July 2021

Citation:

Rodella C, Cespón J, Repetto C and Pellicciari MC (2021) Customized

Application of tDCS for Clinical Rehabilitation in Alzheimer's Disease. Front. Hum. Neurosci. 15:687968. doi: 10.3389/fnhum.2021.687968 cognitive functioning in healthy and pathological aging (Hsu et al., 2015; Cappon et al., 2016), and to integrate this modality in dementia rehabilitation programs (Prehn and Flöel, 2015). In particular, some experimental (Ferrucci et al., 2008; Boggio et al., 2012; Marceglia et al., 2016) and meta-analytical (Hill et al., 2016; Indahlastari et al., 2021) studies have supported the clinical utility of transcranial direct current stimulation (tDCS). The potential of tDCS lies on its mechanisms of action. Specifically, tDCS has local impacts on the GABA/glutamate balance (Stagg et al., 2009), which has been found to be altered in AD patients (Guerra et al., 2011). It also influences functional connectivity, synchronization, and oscillatory activities in prefrontal cortex (Keeser et al., 2011), a region substantially affected by $\mathrm{AD}$. In addition, tDCS may have non-neuronal effects, as almost all tissues and cells are sensitive to electric fields (Ruohonen and Karhu, 2012). For instance, tDCS could potentially modulate the inflammatory response and the conformation of beta-amyloid and other pathological proteins (Toschi et al., 2009), involved in the progression of AD.

Nevertheless, several studies have found very little or null effects (e.g., Horvath et al., 2015) of tDCS on various cognitive domains. These results have been attributed to high inter-study (Pellicciari and Miniussi, 2018) and inter-individual variability in response to tDCS (Li et al., 2015), even though the sources of inter-individual variability were not clearly identified. Importantly, although the sheer number of parameters (e.g., polarity, intensity, location, electrodes size) that can be varied could represent a weakness of these protocols, they also mean the application of tDCS is highly customizable. Consequently, exploring inter-individual differences and how these might influence the effects of tDCS has become crucial. In the following section, we point to Brain Reserve (and the related grade of brain atrophy), Cognitive Reserve and baseline performance measures as potential sources for the inter-individual variability reflected in results obtained after applying tES protocols.

\section{SOURCES OF INTER-INDIVIDUAL VARIABILITY: BRAIN AND COGNITIVE RESERVE}

Cognitive aging is characterized by high inter-individual variability. The concept of reserve explains why there are different pathways in aging; some individuals may possess resources that allow them 
to mitigate physiological cognitive impairments or to prevent or delay potential neuropathologies (Stern et al., 2019). Brain Reserve (BR) specifically refers to neuroanatomic resources, such as the number of neurons and synapses, that allow a person to maintain cognitive function despite significant loss of neural material substrate (Satz, 1993). By contrast, Cognitive Reserve (CR) explains why individuals with the same brain damage exhibit different clinical outcomes, cognitive performances, and rates of recovery (Stern, 2009). CR is usually enhanced by experiences occurring before the onset of neural decline and is measured using proxy variables, such as education level and occupational status (Barulli and Stern, 2013). Several studies have demonstrated that individuals with high CR exhibit greater neural capacity to cope with structural damage, through more efficient deployment of functional compensatory mechanisms, such as strengthened functional connectivity (Serra et al., 2017). For this reason, high-CR individuals may suffer a higher degree of pathology before they begin to exhibit clinical symptoms of AD (Arenaza-Urquijo et al., 2015) and AD neuropathological markers may be evident in absence of clinical symptoms (Jansen et al., 2015). Robertson (2014) proposed a model of CR, which posits a positive correlation between CR and the presence of a right-lateralized fronto-parietal network, due to compensatory functional re-organization of brain areas. In other words, higher CR promotes bilateral activation during cognitive processes typically lateralized in younger adults, as a function of the re-allocation of brain resources that allow healthy elderly to compensate for physiological decline (Cabeza, 2002). Brosnan et al. (2017) tested this model on visual information processing in elderly subjects, applying anodal tDCS over the right prefrontal cortex, to enhance cortical excitability, and consequently facilitate compensatory mechanisms. Results showed that older adults with lower levels of CR reported improved performance in the left but not right items, such that tDCS temporarily altered their processing speed asymmetry, allowing them to perform at the level of their high-CR peers. Faster processing speeds have been associated with a greater neural efficiency (Speer and Soldan, 2015), due not only to functional, but also to structural differences in high-CR individuals, including, for instance, cortical thickness (Menardi et al., 2018). Thus, CR and BR could potentially be related, in countering brain atrophy, which characterizes physiological and, above all, pathological aging. However, in AD patients this link is not always evident, as higher levels of CR can promote compensatory mechanisms even in the presence of relevant brain damage.

For this reason, both BR and CR should be taken into account when applying tDCS; a certain level of gray matter preservation is needed to obtain beneficial effects from stimulation (Thibaut et al., 2015), and CR levels have been shown to influence the effects of tDCS (Berryhill and Jones, 2012). Nevertheless, controlling for the degree of atrophy or CR status is not a common routine in tDCS studies, which often enroll patients with different levels of pathology and they incorrectly consider them to form part of a homogeneous sample.

Also, modeling studies (Mahdavi and Towhidkhah, 2018) have suggested that the level of reduction of gray matter influences the level of current density induced in the brain by tDCS. This is due to the concurrent increase in both cerebrospinal fluid volume and gray matter atrophy. With the progression of illness, the distance between the scalp, where stimulation electrodes are applied, and the cerebral cortex increases with a consequent decrease in the electrical field generated in the targeted areas. Based on these data, it could be hypothesized that tDCS parameters (e.g., current intensity or the size of electrodes) should be customized to accommodate patients with different grades of brain atrophy.

Levels of CR represent an important inter-individual difference that can also impact cognitive performance. More efficient neural/network functioning, typical of high-CR people, could influence individual cognitive ability and baseline performance (Stern, 2009), even in the presence of similar level of brain damage. Recent studies (Heinen et al., 2016; Hsu et al., 2016) have demonstrated how this variable may be crucial when a neuromodulation protocol is applied. For example, Heinen and co-workers (2016) observed opposite effects when the same tDCS polarity was applied to high and low performers during a working memory task. More specifically, cathodal tDCS resulted in impaired working memory for high but improved working memory in low performers. Other research has also shown that tDCS has a greater effect on memory and attention in low compared to high performers (e.g., Learmonth et al., 2015; Hsu et al., 2016). Nevertheless, the direction of such effects is not always consistent. Others studies have reported lowered cognitive capacity in low performers after anodal tDCS (Learmonth et al., 2015; Hsu et al., 2016). As mentioned above, a possible explanation for these mixed results could simply be that researchers, especially in the field of cognitive rehabilitation, do not usually account for brain damage.

Taken together, these results suggest that individual BR, CR, and baseline performance could influence stimulation effects. This should encourage researchers to control for or, at least, analyze the interaction between these variables when applying tDCS for neuro-rehabilitative aims (Benwell et al., 2015). For instance, greater brain atrophy could suggest that higher current (e.g., higher intensity, or bigger electrodes designed to affect a wider area) are needed to promote the level of cortical excitability required to activate compensatory mechanisms.

\section{APPLYING TDCS TO AD REHABILITATION: CONTROLLING FOR INTER-INDIVIDUAL VARIABILITY}

It has been reported that online (Dedoncker et al., 2016), and cathodal (Cespón et al., 2019) tDCS enhances neural activity underlying performance more than offline, anodal tDCS in cognitively declined patients, but the reverse has been observed in healthy subjects. These findings highlight that the influence of timing and polarity on tDCS outcomes may also depend on the target sample. However, these results are not definitive, and the role of inter-individual variables remains unclear. Comparing tDCS-induced effects in subgroups of AD patients that differ in terms of physiological markers of $\mathrm{AD}$ progression (e.g., brain atrophy, Dubois et al., 2016) might shed light on which 
stimulation parameters are most appropriate given a patient's physiological characteristics.

Moreover, neuroimaging and neurophysiological measures could guide stimulation, by providing information on a patient's cortical status at both the structural and functional levels. For instance, it is possible to assess brain atrophy in a separate session and use the results to choose the more effective montage in terms of current intensity, as well as electrode placement and dimensions. Otherwise, when the degree of intra-individual variability is high, online assessment (i.e., immediately before or continuously during the stimulation) of neurophysiological features is desirable. Cortical states can vary considerably between sessions and also across the day (Bergmann et al., 2016).

Even though it is not always possible to change stimulation parameters online, constant monitoring of the modifications that occur, for example, in brain oscillations and eventrelated potentials (ERPs-as assessed with EEG) or eventrelated field (ERFs-as assessed with MEG) or in cortical excitability (TMS-EEG co-registration), could lead researchers to find the best parameters for each patient. The rehabilitation protocol could then be adapted based on the results, in terms of neurophysiological and behavioral modifications, obtained after each session or small set of sessions. Adding such neurophysiological measures to a patient profile might be particularly useful when behavioral outcomes are not sensitive enough to reveal physiological modifications. This intuition has been pointed out by Bergmann et al. (2016), who have shown the potential advantages of neuroimaging and electrophysiological approaches in guiding neurostimulation, by suggesting precisely where, when, and how to apply the stimulation.

Furthermore, it would be worthwhile to involve multiple research groups, who could cooperate to characterize subsets of patients. This would mean developing a large-scale openaccess database, collating all the data and research results on the effects (and null-effects) of tDCS in relation to particular subject characteristics. Such knowledge could then orientate future rehabilitation programs, and help researchers to overcome their own limitations. Indeed, the difficulty of controlling all the variables (e.g., degree of atrophy, which would require access to an MRI scanner; degree of CR, which cannot be directly measured but estimated through proxy variables) that could intervene when tDCS is applied for clinical purpose could

\section{REFERENCES}

Arenaza-Urquijo, E. M., Wirth, M., and Chételat, G. (2015). Cognitive reserve and lifestyle: moving towards preclinical Alzheimer's disease. Front. Aging Neurosci. 7:134. doi: 10.3389/fnagi.2015.00134

Barulli, D., and Stern, Y. (2013). Emerging concepts in cognitive reserve. Trends Cogn. Sci. 17, 1-17. doi: 10.1016/j.tics.2013.08.012

Benwell, C. S. Y., Learmonth, G., Miniussi, C., Harvey, M., and Thut, G. (2015). Non-linear effects of transcranial direct current stimulation as a function of individual baseline performance: evidence from biparietal tDCS influence on lateralized attention bias. Cortex 69, 152-165. doi: 10.1016/j.cortex.2015.05.007

Bergmann, T. O., Karabanov, A., Hartwigsen, G., Thielscher, A., and Siebner, H. R. (2016). Combining non-invasive transcranial brain stimulation represent the reason why it is not common to customize the intervention on patients' differences. A starting point in this direction is the Center for Open Science (COS), a nonprofit technology and cultural exchange company, which has created a powerful online tool, the Open Science Framework. Their goal is to offer the scientific community a platform to share knowledge, ideas, research questions, results, and hypotheses (Miguel et al., 2014; www.cos.io). Research should be focused on analyzing the diversity instead of the similarities among patients, leading to profound collaboration among the research and clinical communities, in order to promote robust studies able to characterize subsets of patients.

\section{CONCLUSIONS}

The high variability among $\mathrm{AD}$ patients makes it difficult to identify the best NIBS protocols. Understanding how interactions between $\mathrm{BR}, \mathrm{CR}$, and performance levels may influence outcomes and how these variables can be controlled by researchers should represent a first step. With this aim, integration of brain stimulation protocols and neuroimaging/neurophysiology measures will be needed to develop customized rehabilitation programs. Structural and functional information on patients' cortical status could guide stimulation by pinpointing the appropriate (1) target area(s) and/or electrode orientation(s); (2) best stimulation onset times; and (3) tDCS parameters (e.g., intensity, polarity, wave form), and by (4) providing online or offline feedback about how brain states are changed by the stimulation.

\section{AUTHOR CONTRIBUTIONS}

CRo, JC, and MP contributed to conception of the opinion. CRo wrote the first draft of the manuscript. All authors contributed to manuscript revision, read, and approved the submitted version.

\section{FUNDING}

This work was supported by the Basque Government through the BERC 2018-2021 program and by the Spanish State Research Agency through BCBL Severo Ochoa excellence accreditation SEV-2015-0490 for an English revision of the manuscript. with neuroimaging and electrophysiology: current approaches and future perspectives. Neuroimage 140, 4-19. doi: 10.1016/j.neuroimage.2016.02.012

Berryhill, M. E., and Jones, K. T. (2012). tDCS selectively improves working memory in older adults with more education. Neurosci. Lett. 521, 148-151. doi: 10.1016/j.neulet.2012.05.074

Boggio, P. S., Ferrucci, R., Mameli, F., Martins, D., Martins, O., Vergari, M., et al. (2012). Prolonged visual memory enhancement after direct current stimulation in Alzheimer's disease. Brain Stimul. 5, 223-230. doi: 10.1016/j.brs.2011.06.006

Brosnan, M. B., Demaria, G., Petersen, A., Dockree, P. M., Robertson, I. H., and Wiegand, I. (2017). Plasticity of the right-lateralized cognitive reserve network in ageing. Cereb. Cortex 28, 1749-1759. doi: 10.1093/cercor/bhx085

Cabeza, R. (2002). Hemispheric asymmetry reduction in older adults: the HAROLD model. Psychol. Aging 17, 85-100. doi: 10.1037/0882-7974.17.1.85 
Cappon, D., Jahanshahi, M., and Bisiacchi, P. (2016). Value and efficacy of transcranial direct current stimulation in the cognitive rehabilitation : a critical review since 2000. Front. Neurosci. 10:157. doi: 10.3389/fnins.2016.00157

Cass, S. P. (2017). Alzheimer's disease and exercise: a literature review. Curr. Sports Med. Rep. 16, 19-22. doi: 10.1249/JSR.0000000000000332

Cespón, J., Miniussi, C., and Pellicciari, M. C. (2018). Interventional programmes to improve cognition during healthy and pathological ageing: cortical modulations and evidence for brain plasticity. Ageing Res. Rev. 43, 81-98. doi: 10.1016/j.arr.2018.03.001

Cespón, J., Rodella, C., Miniussi, C., and Pellicciari, M. C. (2019). Behavioural and electrophysiological modulations induced by transcranial direct current stimulation in healthy elderly and Alzheimer's disease patients: a pilot study. Clinic. Neurophysiol. 130, 2038-2052. doi: 10.1016/j.clinph.2019.08.016

Dedoncker, J., Brunoni, A. R., Baeken, C., and Vanderhasselt, M.-A. (2016). A systematic review and meta-analysis of the effects of transcranial direct current stimulation (tDCS) over the dorsolateral prefrontal cortex in healthy and neuropsychiatric samples: influence of stimulation parameters. Brain Stimul. 9, 1-17. doi: 10.1016/j.brs.2016.04.006

Dubois, B., Hampel, H., Feldman, H. H., Scheltens, P., Aisen, P., Andrieu, S., et al. (2016). Preclinical Alzheimer's disease: definition, natural history, and diagnostic criteria. Alzheimers Dement. 12, 292-323. doi: $10.1016 /$ j.jalz.2016.02.002

Ferrucci, R., Mameli, F., Guidi, I., Mrakic-Sposta, S., Vergari, M., Marceglia, S., et al. (2008). Transcranial direct current stimulation improves recognition memory in Alzheimer disease. Neurology 71, 493-498. doi: 10.1212/01.wnl.0000317060.43722.a3

Guerra, A., Assenza, F., Bressi, F., Scrascia, F., Del Duca, M., Ursini, F., et al. (2011). Transcranial magnetic stimulation studies in Alzheimer's disease. Int. J. Alzheimer's Dis. 2011:263817. doi: 10.4061/2011/263817

Heinen, K., Sagliano, L., Candini, M., Husain, M., Cappelletti, M., and Zokaei, N. (2016). Cathodal transcranial direct current stimulation over posterior parietal cortex enhances distinct aspects of visual working memory. Neuropsychologia 87, 35-42. doi: 10.1016/j.neuropsychologia.2016.04.028

Hill, A. T., Fitzgerald, P. B., and Hoy, K. E. (2016). Effects of anodal transcranial direct current stimulation on working memory: a systematic review and metaanalysis of findings from healthy and neuropsychiatric populations. Brain Stimul. 9, 197-208. doi: 10.1016/j.brs.2015.10.006

Horvath, J. C., Forte, J. D., and Carter, O. (2015). Quantitative review finds no evidence of cognitive effects in healthy populations from single-session transcranial direct current stimulation (tDCS). Brain Stimul. 8, 535-550. doi: 10.1016/j.brs.2015.01.400

Hsu, T.-Y., Juan, C.-H., and Tseng, P. (2016). Individual differences and statedependent responses in transcranial direct current stimulation. Front. Hum. Neurosci. 10:643. doi: 10.3389/fnhum.2016.00643

Hsu, W.-Y., Ku, Y., Zanto, T. P., and Gazzaley, A. (2015). Effects of non-invasive brain stimulation on cognitive function in healthy aging and Alzheimer's disease: a systematic review and meta-analysis. Neurobiol. Aging 36, 2348-2359. doi: 10.1016/j.neurobiolaging.2015.04.016

Indahlastari, A., Hardcastle, C., Albizu, A., Alvarez-Alvarado, S., Boutzoukas, E. M., Evangelista, N. D., et al. (2021). A systematic review and meta-analysis of transcranial direct current stimulation to remediate age-related cognitive decline in healthy older adults. Neuropsychiatr. Dis. Treat. 17, 971-990. doi: 10.2147/NDT.S259499

Jansen, W. J., Ossenkoppele, R., Knol, D. L., Tijms, B. M., Scheltens, P., Verhey, F. R. J., et al. (2015). Prevalence of cerebral amyloid pathology in persons without dementia. JAMA 313:1924. doi: 10.1001/jama.2015.4668

Keeser, D., Padberg, F., Reisinger, E., Pogarell, O., Kirsch, V., Palm, U., et al. (2011). Prefrontal direct current stimulation modulates resting EEG and event-related potentials in healthy subjects: a standardized low resolution tomography (sLORETA) study. Neuroimage 55, 644-657. doi: 10.1016/j.neuroimage.2010.12.004

Learmonth, G., Thut, G., Benwell, C. S. Y., and Harvey, M. (2015). The implications of state-dependent tDCS effects in aging: behavioural response is determined by baseline performance. Neuropsychologia 74, 108-119. doi: 10.1016/j.neuropsychologia.2015.01.037

Li, L. M., Uehara, K., and Hanakawa, T. (2015). The contribution of interindividual factors to variability of response in transcranial direct current stimulation studies. Front. Cellular Neurosci. 9:181. doi: 10.3389/fncel.2015. 00181
Mahdavi, S., and Towhidkhah, F. (2018). Computational human head models of tDCS: Influence of brain atrophy on current density distribution. Brain Stimul. 11, 104-107. doi: 10.1016/j.brs.2017.09.013

Marceglia, S., Mrakic-Sposta, S., Rosa, M., Ferrucci, R., Mameli, F., Vergari, M., et al. (2016). Transcranial direct current stimulation modulates cortical neuronal activity in Alzheimer's disease. Front. Neurosci. 10, 1-11. doi: $10.3389 /$ fnins.2016.00134

Menardi, A., Pascual-Leone, A., Fried, P. J., and Santarnecchi, E. (2018). The role of cognitive reserve in Alzheimer's disease and aging: a multi-modal imaging review. J. Alzheimer's Dis. 66, 1341-1362. doi: 10.3233/JAD-180549

Miguel, E., Camerer, C., Casey, K., Cohen, J., Esterling, K. M., Gerber, A., et al. (2014). Promoting transparency in social science research. Science 343, 30-31. doi: 10.1126/science.1245317

Pellicciari, M. C., and Miniussi, C. (2018). Transcranial direct current stimulation in neurodegenerative disorders. J. ECT 34, 193-202. doi: 10.1097/YCT.0000000000000539

Prehn, K., and Flöel, A. (2015). Potentials and limits to enhance cognitive functions in healthy and pathological aging by tDCS. Front. Cell. Neurosci. 9:355. doi: 10.3389/fncel.2015.00355

Robertson, I. H. (2014). A right hemisphere role in cognitive reserve. Neurobiol. Aging 35, 1375-1385. doi: 10.1016/j.neurobiolaging.2013.11.028

Ruohonen, J., and Karhu, J. (2012). TDCS possibly stimulates glial cells. Clinic. Neurophysiol. 123, 2006-2009. doi: 10.1016/j.clinph.2012.02.082

Satz, P. (1993). Brain reserve capacity on symptom onset after brain injury: a formulation and review of evidence for threshold theory. Neuropsychology 7 , 273-295. doi: 10.1037/0894-4105.7.3.273

Serra, L., Mancini, M., Cercignani, M., Di Domenico, C., Span,ò, B., Giulietti, G., et al. (2017). Network-based substrate of cognitive reserve in Alzheimer's disease. J. Alzheimer's Dis. 55, 421-430. doi: 10.3233/JAD-160735

Speer, M. E., and Soldan, A. (2015). Cognitive reserve modulates ERPs associated with verbal working memory in healthy younger and older adults. Neurobiol. Aging 36, 1424-1434. doi: 10.1016/j.neurobiolaging.2014.12.025

Stagg, C. J., Best, J. G., Stephenson, M. C., O’Shea, J., Wylezinska, M., Kincses, Z. T., et al. (2009). Polarity-sensitive modulation of cortical neurotransmitters by transcranial stimulation. J. Neurosci. 29, 5202-5206. doi: 10.1523/JNEUROSCI.4432-08.2009

Stern, Y. (2009). Cognitive reserve. Neuropsychologia 47, 2015-2028. doi: 10.1016/j.neuropsychologia.2009.03.004

Stern, Y., Barnes, C. A., Grady, C. L., Jones, R. N., and Raz, N. (2019). Brain reserve, cognitive reserve, compensation, and maintenance: operationalization, validity, and mechanisms of cognitive resilience. Neurobiol. Aging 83, 124-129. doi: 10.1016/j.neurobiolaging.2019.03.022

Thibaut, A., Di Perri, C., Chatelle, C., Bruno, M. A., Bahri, M. A., Wannez, S., et al. (2015). Clinical response to tDCS depends on residual brain metabolism and grey matter integrity in patients with minimally conscious state. Brain Stimul. 8, 1116-1123. doi: 10.1016/j.brs.2015.07.024

Toschi, F., Lugli, F., Biscarini, F., and Zerbetto, F. (2009). Effects of electric field stress on a $\beta$-amyloid peptide. J. Physic. Chem. B 113, 369-376. doi: $10.1021 /$ jp807896g

Weintraub, S., Wicklund, A. H., and Salmon, D. P. (2012). The neuropsychological profile of alzheimer disease. Cold Spring Harbor Prespect. Med. 4:a00617. doi: $10.1101 /$ cshperspect.a006171

Conflict of Interest: The authors declare that there are no commercial or financial relationships that could be construed as a potential conflict of interest.

Publisher's Note: All claims expressed in this article are solely those of the authors and do not necessarily represent those of their affiliated organizations, or those of the publisher, the editors and the reviewers. Any product that may be evaluated in this article, or claim that may be made by its manufacturer, is not guaranteed or endorsed by the publisher.

Copyright (c) 2021 Rodella, Cespón, Repetto and Pellicciari. This is an open-access article distributed under the terms of the Creative Commons Attribution License (CC $B Y)$. The use, distribution or reproduction in other forums is permitted, provided the original author(s) and the copyright owner(s) are credited and that the original publication in this journal is cited, in accordance with accepted academic practice. No use, distribution or reproduction is permitted which does not comply with these terms. 